\title{
Validez y consistencia de una escala rápida de satisfacción del paciente de consulta externa
}

\author{
Arturo García-Galicia, ${ }^{1}$ José Francisco Díaz-Díaz, ${ }^{2}$ Álvaro José Montiel-Jarquín, ${ }^{3 *}$ \\ Akihiki Mizuki González-López, ${ }^{4}$ Eduardo Vázquez-Cruz y Carlos Francisco Morales-Flores ${ }^{1}$ \\ ${ }^{1}$ Centro Médico Nacional "General de División Manuel Ávila Camacho", Hospital de Traumatología y Ortopedia; ${ }^{2}$ Hospital General de Zona 20; \\ ${ }^{3}$ Centro Médico Nacional "General de División Manuel Ávila Camacho", Hospital de Especialidades; ${ }^{4}$ Unidad de Medicina Familiar 1; ${ }^{5}$ Unidad de \\ Medicina Familiar 6. Instituto Mexicano del Seguro Social, Puebla, México
}

\section{Resumen}

Antecedentes: La satisfacción del usuario es clave para definir y valorar la calidad de la atención, sin embargo, no existe una escala rápida de satisfacción del paciente en México. El objetivo fue determinar la validez y consistencia de la Escala Rápida de Satisfacción del Paciente de Consulta Externa (ERSaPaCE). Método: Estudio comparativo, observacional, transversal, prolectivo. En la fase 1 se elaboró un modelo de escala rápida, que se sometió a la valoración de expertos en atención médica; se realizaron pruebas piloto con 10 pacientes por ronda, tantas veces como fuera necesario hasta lograr 20 aprobaciones. En la fase 2 se aplicó el cuestionario resultante y la escala de Satisfacción del Usuario de Consultas Externas (SUCE) a usuarios de consulta externa; la ERSaPaCE se reaplicó telefónicamente siete a 10 días después. Se utilizó estadística descriptiva, $\alpha$ de Cronbach, Spearman y coeficiente de correlación intraclase (CCI). Resultados: Se reclutaron 200 pacientes, 53 \% con edad de 31 a 60 años, $51.5 \%$ mujeres y $48.5 \%$ hombres de la consulta externa de 13 especialidades; $\alpha$ de Cronbach de ERSaPaCE $=0.608, \mathrm{CCl}=0.98(p=0.000)$ y validez convergente $=0.681(p=0.000)$ por rho de Spearman. Conclusiones: $E R S a P a C E$ fue un instrumento válido y consistente para evaluar la satisfacción del usuario de consulta externa.

PALABRAS CLAVE: Validez. Consistencia. Calidad de la atención. Satisfacción del paciente. SUCE. ERSaPaCE.

\section{Validity and consistency of an outpatient department user satisfaction rapid scale}

\section{Abstract}

Background: User satisfaction is key to define and assess the quality of care; however, there is no patient satisfaction rapid scale in Mexico. Our objective was to determine the validity and consistency of an outpatient department user satisfaction rapid scale (ERSaPaCE). Method: Comparative, observational, cross-sectional, prolective study. In phase 1, a rapid scale model was developed, which was submitted to experts in medical care for assessment; the instrument was pilot-tested in 10-patient groups, using as many rounds as required until it obtained 20 approvals. In phase 2, the resulting questionnaire and the Outpatient Service User Satisfaction (SUCE) scale were applied to outpatient department users. ERSaPaCE was reapplied by telephone 10 days later. Descriptive statistics, Cronbach's $\alpha$, Spearman's correlation and intra-class correlation coefficient (ICC) were used. Results: Two-hundred patients were recruited, out of which $53 \%$ were aged 31-60 years; $51.5 \%$ were women and $48.5 \%$ men, all of them users of the outpatient services from 13 specialties. Cronbach's $\alpha$ for ERSaPaCE was 0.608, whereas ICC was $0.98(p=0.000)$. Convergent validity was $0.681(p=0.000)$ using Spearman's rho. Conclusion: ERSaPaCE was a valid and consistent instrument for the assessment of outpatient department user satisfaction.

KEY WORDS: Validity. Consistency. Quality of care. Patient satisfaction. SUCE. ERSaPaCE.

Correspondencia:

*Álvaro José Montiel-Jarquín

E-mail: dralmoja @ hotmail.com

0016-3813/@ 2019 Academia Nacional de Medicina de México, A.C. Publicado por Permanyer. Éste es un artículo open access bajo la licencia CC BY-NC-ND (http://creativecommons.org/licenses/by-nc-nd/4.0/).
Fecha de recepción: 07-03-2019

Fecha de aceptación: 21-06-2019

875/GMM.19005144
Gac Med Mex. 2020;156:47-52

Disponible en PubMed

remexico.com 


\section{Introducción}

La calidad en la atención médica se ha transformado en un requisito fundamental en los servicios de salud. Si bien los avances científico-tecnológicos han tenido gran impacto en mejorar la cantidad y calidad de vida de muchos pacientes, también ha generado numerosos problemas con consecuencias negativas, que afectan de una u otra forma la atención en salud. ${ }^{1}$ Por otra parte, la convergencia de administradores y profesionales en el campo de salud, el incremento en los costos y utilización de los servicios por parte de la población, así como la exigencia de los mismos obligan a buscar estándares para cumplir al máximo las expectativas de los pacientes. ${ }^{2}$

En México se cuentan con diversos antecedentes, desde la edición en 1962 de Auditoría médica, por el Instituto Mexicano de Seguro Social, y sus actualizaciones en 1972 y 1973, con énfasis en el expediente clínico. En 1981, Donabedian inició la evaluación de la calidad de la atención médica propiamente dicha con base en la teoría de sistemas. Finalmente, desde 1999 la Secretaría de Salud ha diseñado un modelo de certificación de hospitales para mejorar la atención a la salud en México. ${ }^{3-7}$

La calidad de la atención médica puede definirse como "otorgar atención médica al paciente oportunamente, con competencia profesional, seguridad y respeto a los principios éticos, con el propósito de satisfacer sus necesidades de salud y expectativas"., 7

La satisfacción de los usuarios externos expresa un juicio de valor individual y subjetivo, clave en la definición y valoración de la calidad en la atención, por lo que es un indicador para evaluar la calidad de atención en los servicios de salud. ${ }^{5,6}$ Este constructo incluye amabilidad en el trato, disponibilidad de equipos, material, insumos y medicamentos necesarios, personal suficiente y calificado, continuidad en el proceso de atención, comodidad en las áreas de servicio y costos razonables. ${ }^{8,9}$

De esta manera, las encuestas de satisfacción de los usuarios externos requieren propiedades psicométricas que garanticen su fiabilidad y validez, por lo tanto, es necesario contar con encuestas validadas y de fácil aplicación para medir el nivel de satisfacción e identificar las principales causas de insatisfacción de los usuarios externos en servicios de salud, que permitan implementar acciones de mejora. ${ }^{8-10}$ Por otra parte, dado que la satisfacción es un constructo subjetivo, es preferible utilizar escalas de autorreporte, consideradas el estándar de criterio en este tipo de variables. ${ }^{11,12}$

Si bien existen diversos reportes de satisfacción del usuario, ${ }^{13-15}$ en México no se cuenta con una escala rápida de evaluación de la satisfacción del usuario en establecimientos de segundo nivel de atención de salud. Por tal motivo, creemos necesario desarrollar un instrumento que coadyuve a someter a la institución o servicio de salud a un programa de mejora y así ofertar una atención de calidad que cumpla con las expectativas de pacientes y familiares.

El instrumento que se pretende diseñar debe ser una escala rápida, funcional, de autorreporte (de ser posible), para que el paciente no invierta mucho tiempo o esfuerzo en su respuesta y que al mismo tiempo pruebe ser válido y consistente. El objetivo de este estudio fue diseñar una nueva escala rápida de satisfacción del usuario de consulta externa de segundo nivel y determinar su validez y consistencia.

\section{Método}

Estudio comparativo, observacional, prolectivo, homodémico, longitudinal, en pacientes atendidos en consulta externa del Hospital General de Zona 20 del Instituto Mexicano de Seguro Social en Puebla, a su salida del servicio. Fue aprobado por el Comité Local de Investigación y Ética en Investigación en Salud 2102 del Instituto Mexicano de Seguro Social (registro R-2013-2102-20). Se incluyeron pacientes de uno y otro sexo, sin importar su edad, que aceptaron contestar la encuesta. Se eliminaron los sujetos que no completaron el cuestionario.

\section{Fase 1. Elaboración de la escala}

Se elaboró un modelo de escala rápida (por un comité de expertos: un psicólogo asesor de calidad, un pediatra experto en calidad y clinimetría y un experto en consulta externa), que posteriormente se sometió a la evaluación de diferentes expertos en el área (un maestro en administración de hospitales, el director de la unidad y dos supervisores de calidad de atención hospitalaria). La escala se sometió a un estudio piloto con los usuarios para determinar claridad en la redacción, facilidad de la respuesta y rapidez y practicidad; se realizaron modificaciones en estos aspectos sin afectar la opinión de los expertos. El estudio piloto se repitió tantas veces (10 pacientes por ronda) como fue necesario hasta lograr la aprobación total de 20 pacientes. 


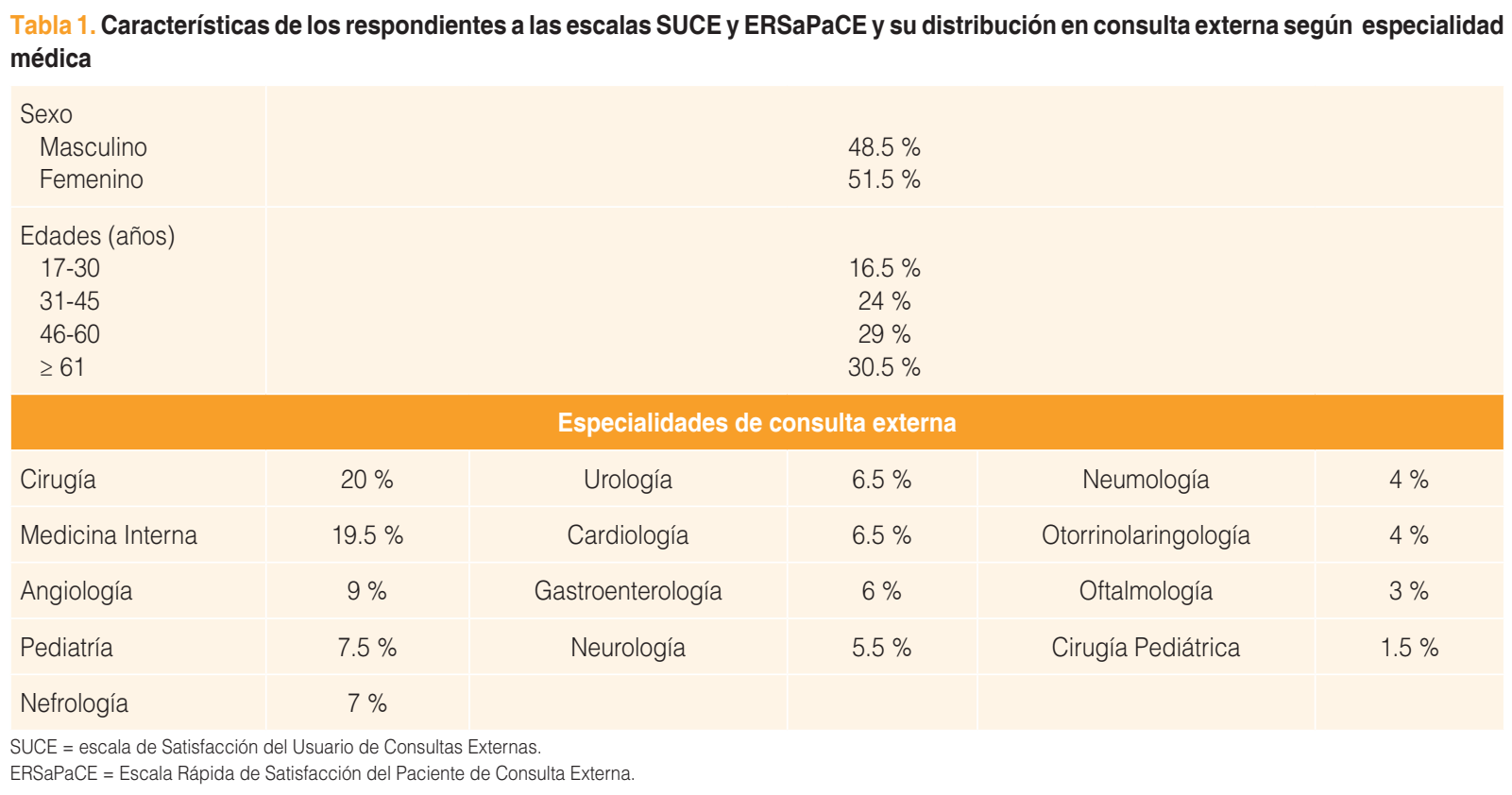

\section{Fase 2. Fase de validez y consistencia}

En un periodo de seis meses, a todos los usuarios de consulta externa se les aplicó el cuestionario resultante de la fase 1 (ERSaPaCE, Escala Rápida de Satisfacción del Paciente de Consulta Externa) y la escala de Satisfacción del Usuario de Consultas Externas (SUCE)..$^{10}$ En cada caso, la ERSaPaCE se reaplicó vía telefónica entre siete y 10 días después.

Se solicitó consentimiento informado en forma escrita a los usuarios del servicio de consulta externa; se evitó cualquier intervención que pudiera condicionar perjuicio al paciente y se guardó la confidencialidad de los datos para evitar posibles consecuencias negativas en la atención médica en la unidad.

Se utilizó estadística descriptiva. Para la valoración de la consistencia interna se utilizó $\alpha$ de Cronbach; para la validez convergente de las escalas, rho de Spearman; y para prueba-reprueba de la escala rápida, coeficiente de correlación intraclase $(\mathrm{CCl}){ }^{16}$

\section{Resultados}

\section{Fase 1}

La escala diseñada y aprobada por los expertos requirió tres rondas piloto hasta que la redacción de las preguntas fue aceptada por los usuarios. Para mejorar la aplicabilidad y reforzar el entendimiento de los ítems, en cada valor de las respuestas se optó por incluir los dibujos de caras utilizados en una escala facial del dolor, ${ }^{11}$ cuya validez de constructo se demostró ampliamente. Esta fase finalizó con la conclusión de la escala, como se muestra en la Figura 1.

\section{Fase 2}

Se encuestaron 200 pacientes usuarios de la consulta externa de los diferentes servicios, cuyas características se detallan en la Tabla 1. No hubo pacientes eliminados del estudio. En los menores de edad, SUCE y ERSaPaCE se aplica a los padres o adultos acompañantes.

El resultado al aplicar $\alpha$ de Cronbach a ERSaPaCE fue de 0.608 y el del coeficiente de correlación intraclase para la concordancia prueba-reprueba fue de $0.98(p=0.000)$ La correlación de ambas escalas por coeficiente de Spearman fue de $0.681(p=0.000)$. Los resultados de ambas escalas se muestran en la Tabla 2.

En la ERSaPaCE se incluyó un rubro para las felicitaciones, sugerencias y quejas; textualmente las más frecuentes fueron las siguientes:

- Mejorar la atención en el archivo clínico, por favor.

- Aseo más frecuente en los baños; no existen toallas desechables ni papel.

- Que el tratante cambie su actitud.

- Que el médico no explica mi padecimiento, es prepotente y me gritó.

- Por favor, que surtan medicamentos en farmacia. 


\section{Escala Rápida de Satisfacción de Consulta Externa (ERSaPaCE)}

Nombre (opcional):

Teléfono (opcional):

Consulta a la que acudió:

APRECIADO DERECHOHABIENTE: para fines de calificación de la calidad de la atención le pedimos respetuosamente nos responda la siguiente encuesta. Agradecemos su comprensión y sinceridad. Sus respuestas serán confidenciales.

\begin{tabular}{|c|c|c|c|c|c|}
\hline \multirow{2}{*}{$\begin{array}{l}\text { INSTRUCCIONES: Tache con una "X" la } \\
\text { respuesta que elija }\end{array}$} & \multicolumn{5}{|c|}{ PARÁMETROS } \\
\hline & 1 & 2 & 3 & 4 & 5 \\
\hline ¿Cómo califica... & 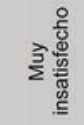 & & & 崖 & \\
\hline 1. La limpieza y el orden de la Unidad Médica? & & & & & \\
\hline 2. El trato personal por parte de la Asistente Médica? & & & & & \\
\hline $\begin{array}{l}\text { 3. El trato, respeto atención y disponibilidad por parte del } \\
\text { personal de Enfermería? }\end{array}$ & & & & & \\
\hline $\begin{array}{l}\text { 4. El trato, respeto atención y disponibilidad por parte del } \\
\text { Médico Titular o Tratante? }\end{array}$ & & & & & \\
\hline $\begin{array}{l}\text { 5. Información por parte del Médico sobre el estado de } \\
\text { salud del paciente? }\end{array}$ & & & & & \\
\hline $\begin{array}{l}\text { 6. La atención de la ventanilla de Archivo Clínico para su } \\
\text { expediente? }\end{array}$ & & & & & \\
\hline 7. La atención de la Farmacia para surtir su receta? & & & & & \\
\hline $\begin{array}{l}\text { 8. La imagen institucional del Médico, Enfermera y } \\
\text { Asistente Médica? }\end{array}$ & & & & & \\
\hline $\begin{array}{l}\text { 9. El tiempo en la sala de espera para su atención } \\
\text { médica? }\end{array}$ & & & & & \\
\hline
\end{tabular}

10. De acuerdo con sus respuestas anteriores, la unidad médica debería:

a. Recibir felicitación(es ) por su valioso y efectivo desempeño

b. Recibir sugerencia(s) para mejorar el servicio médico

c. Recibir queja(s) para expresar su inconformidad del servicio médico

Por favor escriba la felicitación, sugerencia o queja:

\begin{tabular}{|l|}
\hline \\
\hline
\end{tabular}

Figura 1. Escala rápida de satisfacción del paciente de consulta externa.

- Los policías son prepotentes y mal educados.

- Felicidades (a tres diferentes médicos tratantes).

\section{Discusión}

Dado que la satisfacción del paciente es útil, si bien en forma indirecta, para determinar la calidad de la atención, se registran diversos esfuerzos para evaluarla desde diferentes ángulos y con profundidad variada. $6,8-10,13-20$

En este estudio se presenta una escala de satisfacción del usuario de consulta externa del área hospitalaria, que puede ser aplicada en cualquier unidad médica o área de atención ambulatoria de 
Tabla 2. Comparación de resultados de SUCE y ERSaPaCE

\begin{tabular}{|c|c|c|c|}
\hline \multicolumn{2}{|c|}{ Escala SUCE } & \multicolumn{2}{|l|}{ ERSaPaCE } \\
\hline \multicolumn{2}{|c|}{$\begin{array}{l}\text { La comodidad de la sala } \\
\text { de espera }\end{array}$} & \multicolumn{2}{|c|}{$\begin{array}{l}\text { Limpieza y orden de la unidad } \\
\text { médica }\end{array}$} \\
\hline $\begin{array}{l}1 \text { punto } \\
4 \text { puntos } \\
5 \text { puntos } \\
6 \text { puntos } \\
7 \text { puntos } \\
8 \text { puntos } \\
9 \text { puntos } \\
10 \text { puntos }\end{array}$ & $\begin{array}{c}0.5 \% \\
1 \% \\
3.5 \% \\
2.5 \% \\
7 \% \\
16 \% \\
30 \% \\
39.5 \%\end{array}$ & $\begin{array}{l}\text { Muy insatisfecho } \\
\text { Insatisfecho } \\
\text { Poco satisfecho } \\
\text { Satisfecho } \\
\text { Muy satisfecho }\end{array}$ & $\begin{array}{c}2 \% \\
4 \% \\
17 \% \\
50 \% \\
27 \%\end{array}$ \\
\hline \multicolumn{2}{|c|}{$\begin{array}{l}\text { El trato por parte del } \\
\text { personal de enfermeria }\end{array}$} & \multicolumn{2}{|c|}{$\begin{array}{l}\text { Trato, respeto, atención y } \\
\text { disponibilidad por parte del } \\
\text { personal de enfermeria }\end{array}$} \\
\hline $\begin{array}{l}3 \text { puntos } \\
5 \text { puntos } \\
6 \text { puntos } \\
7 \text { puntos } \\
8 \text { puntos } \\
9 \text { puntos } \\
10 \text { puntos }\end{array}$ & $\begin{array}{c}0.5 \% \\
1 \% \\
2.5 \% \\
4 \% \\
17.5 \% \\
27.5 \% \\
47 \%\end{array}$ & $\begin{array}{l}\text { Muy insatisfecho } \\
\text { Insatisfecho } \\
\text { Poco satisfecho } \\
\text { Satisfecho } \\
\text { Muy satisfecho }\end{array}$ & $\begin{array}{c}0.5 \% \\
6 \% \\
13 \% \\
44.5 \% \\
36 \%\end{array}$ \\
\hline \multicolumn{2}{|c|}{$\begin{array}{l}\text { El trato por parte del } \\
\text { personal médico }\end{array}$} & \multicolumn{2}{|c|}{$\begin{array}{l}\text { Trato, respeto, atención y } \\
\text { disponibilidad por parte del } \\
\text { personal médico }\end{array}$} \\
\hline $\begin{array}{l}1 \text { punto } \\
3 \text { puntos } \\
4 \text { puntos } \\
5 \text { puntos } \\
6 \text { puntos } \\
7 \text { puntos } \\
8 \text { puntos } \\
9 \text { puntos } \\
10 \text { puntos }\end{array}$ & $\begin{array}{c}1 \% \\
2 \% \\
0.5 \% \\
1 \% \\
2.5 \% \\
6 \% \\
12 \% \\
29.5 \% \\
45.5 \%\end{array}$ & $\begin{array}{l}\text { Muy insatisfecho } \\
\text { Insatisfecho } \\
\text { Poco satisfecho } \\
\text { Satisfecho } \\
\text { Muy satisfecho }\end{array}$ & $\begin{array}{c}0 \% \\
7.5 \% \\
13.5 \% \\
34 \% \\
45 \%\end{array}$ \\
\hline \multicolumn{2}{|c|}{$\begin{array}{l}\text { La información recibida } \\
\text { sobre su problema de } \\
\text { salud }\end{array}$} & \multicolumn{2}{|c|}{$\begin{array}{l}\text { Información por parte del médico } \\
\text { sobre su estado de salud }\end{array}$} \\
\hline $\begin{array}{l}3 \text { puntos } \\
4 \text { puntos } \\
5 \text { puntos } \\
6 \text { puntos } \\
7 \text { puntos } \\
8 \text { puntos } \\
9 \text { puntos } \\
10 \text { puntos }\end{array}$ & $\begin{array}{c}0.5 \% \\
1 \% \\
4 \% \\
1.5 \% \\
9 \% \\
12.5 \% \\
31.5 \% \\
40 \%\end{array}$ & $\begin{array}{l}\text { Muy insatisfecho } \\
\text { Insatisfecho } \\
\text { Poco satisfecho } \\
\text { Satisfecho } \\
\text { Muy satisfecho }\end{array}$ & $\begin{array}{c}0.5 \% \\
6 \% \\
13 \% \\
37.5 \% \\
43 \%\end{array}$ \\
\hline \multicolumn{2}{|c|}{$\begin{array}{l}\text { Los trámites que tuvo } \\
\text { que hacer en admisión }\end{array}$} & \multicolumn{2}{|c|}{$\begin{array}{l}\text { Respuesta de la ventanilla de } \\
\text { archivo clínico para su expediente }\end{array}$} \\
\hline $\begin{array}{l}1 \text { punto } \\
3 \text { puntos } \\
4 \text { puntos } \\
5 \text { puntos } \\
6 \text { puntos } \\
7 \text { puntos } \\
8 \text { puntos } \\
9 \text { puntos } \\
10 \text { puntos }\end{array}$ & $\begin{array}{c}1 \% \\
2 \% \\
0.5 \% \\
1.5 \% \\
2 \% \\
7 \% \\
23 \% \\
31.5 \% \\
31.5 \%\end{array}$ & $\begin{array}{l}\text { Muy insatisfecho } \\
\text { Insatisfecho } \\
\text { Poco satisfecho } \\
\text { Satisfecho } \\
\text { Muy satisfecho }\end{array}$ & $\begin{array}{c}2.5 \% \\
9.5 \% \\
12.5 \% \\
40 \% \\
35.5 \%\end{array}$ \\
\hline
\end{tabular}

Tabla 2. Comparación de resultados de SUCE y ERSaPaCE (Continuación)

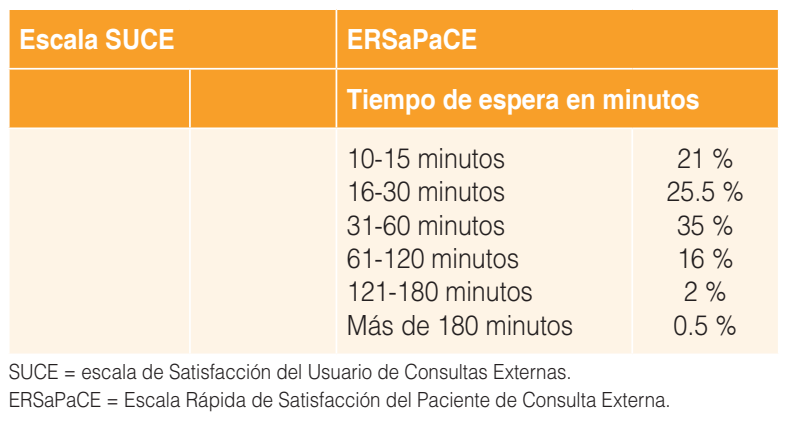

cualquier nivel de atención, además de cumplir con las características de brevedad, rapidez, practicidad para ser contestada; con ella es posible evaluar los diferentes servicios o momentos de la atención ambulatoria.

El procedimiento de la fase 1 del estudio, en el que se involucraron hasta 10 diferentes expertos en el diseño y evaluación, confiere validez de apariencia y criterio al ERSaPaCE. De esta forma, la escala permite obtener una evaluación de los diferentes aspectos de la atención de consulta externa, incluyendo los propios de la unidad médica, así como del personal médico-paramédico y administrativo (archivo clínico), similar a otras escalas, ${ }^{6,10,13-20}$ además, introduce el tiempo de espera (indicador importante en la calidad del servicio) y la oportunidad para que el paciente externe alguna opinión positiva o negativa (felicitación, sugerencias o quejas), acercando al paciente con el otorgador del servicio. Estos dos últimos aspectos innovadores hacen que la escala aporte información muy útil para el administrador de servicios de salud.

La respuesta completa del cuestionario se favorece por la corta extensión: solo consta de 10 ítems de rápida y fácil respuesta y una opinión por escrito (opcional), gran ventaja en relación con otras escalas más extensas, más elaboradas o de respuesta antes y después de la consulta (citas). Todos nuestros pacientes completaron la encuesta, por ello ninguno fue eliminado. Además, como equivalentes a los valores en cada ítem se agregaron las caritas diseñadas por García Galicia et al., que han demostrado gran consistencia y validez de constructo para escalas globales subjetivas. ${ }^{12}$

La participación de diferentes expertos en evaluación de calidad que laboran tanto en el área operativa como en la directiva, quienes se ajustaron a los 
propósitos de rapidez y practicidad, dotó al cuestionario de un alto valor en cuanto a sensatez y validez por apariencia (validez de constructo).

Al evaluar la satisfacción del paciente, la población encuestada se distribuyó equilibradamente en cuanto al sexo, con lo que se evitó el sesgo que pudiera existir por este motivo. En cuanto a la edad de los respondientes, se obtuvo un amplio rango de edades que incluyó adultos jóvenes, maduros y mayores.

Las especialidades y los servicios consultados fueron diversos (13 diferentes especialidades) y en ocasiones más de un médico de alguna especialidad, lo que demuestra la aplicabilidad de las escalas.

ERSaPaCE registró una consistencia interna de 0.608 por $\alpha$ de Cronbach, quizá debido a que consta de pocos ítems (solo 10) y que los aspectos evaluados del proceso de atención ambulatoria fueron también muy variados $y$, presumiblemente, de comportamiento independiente (asistentes, enfermería, médicos, archivo, farmacia, etcétera). Sin embargo, mostró aceptable validez convergente con la SUCE, que en su validación registró 0.8 por la misma prueba. $^{10}$

Dadas las características y temporalidad de nuestro estudio, queda pendiente la exploración de la sensibilidad al cambio, quizá con la comparación de la satisfacción del paciente antes y después de alguna maniobra tendente a modificar el proceso de consulta externa, y así mejorar la calidad de la atención en esta modalidad.

Los hallazgos del presente trabajo nos permiten afirmar que la ERSaPaCE es válida y consistente y constituye una alternativa útil para evaluar la calidad de la atención en las unidades médicas.

\section{Financiamiento}

Los autores no recibieron ayuda o financiación para el presente trabajo.

\section{Bibliografía}

1. Empaire G. Calidad de atención médica y principios éticos. Acta Bioethica. 2010;16:127-132.

2. Fernández-Busso N. Calidad de la atención médica. Programa de Educación a Distancia [en línea]. Argentina: Sociedad Argentina de Calidad en Atención de la Salud; 2019.

3. Aguirre H. Administración de la calidad de la atención médica. Rev Med IMSS. 1997;35:257-264.

4. Aguirre-Gas HG. Sistema ISO 9000 o evaluación de la calidad de la atención médica. Cir Cir. 2008;76:187-196.

5. Aguirre H. Evaluación de la calidad de la atención médica. Expectativas de los pacientes y de los trabajadores de las unidades médicas. Salud Publica Mex. 1990;32:170-180.

6. Vera J, Trujillo A. El efecto de la calidad de vida del servicio en la satisfacción del derechohabiente en instituciones públicas de salud en México. Contaduría y Administración. 2018;63:1-22.

7. Consejo de Salubridad General. Estándares para la Certificación de Hospitales 2012 [en línea]. México: Consejo de Salubridad General; 2013.

8. González-Medécigo LE, Gallardo-Díaz EG. Calidad de la atención médica la diferencia entre la vida o la muerte. Rev Digit Universitaria. 2012;13:1067-1071.

9. Crossing the quality chasm: a new health system for the $21^{\text {st }}$ Century Committee. EE. UU.: Institute of Medicine/Committee on Quality of Health Care in America; 2001.

10. Cabello E, Chirinos JL. Validación y aplicabilidad de encuestas SERVQUAL modificadas para medir la satisfacción de usuarios externos en servicios de salud. Rev Med Hered. 2012;23:88-95.

11. Empaire G. Calidad de atención médica y principios éticos. Acta Bioethica. 2010;16:127-132

12. Ramírez TJ, Nájera P, Nigenda G. Percepción de la calidad de la atención de los servicios de salud en México: perspectiva de los usuarios. Salud Publica Mex. 1998;40:1-10

13. Aguirre-Gas HG, Zavala-Villavicencio JA, Hernández-Torres F, Fajardo-Dolci G. Calidad de la atención médica y seguridad del paciente quirúrgico. Error médico, mala práctica y responsabilidad profesional. Cir Cir 2010;78:456-462.

14. Granado S, Rodríguez C, Olmedo MC, Chacón A, Vigil D, Rodríguez P. Diseño y validación de un cuestionario para evaluar la satisfacción de los pacientes atendidos en las consultas externas de un hospital de Madrid en 2006. Rev Esp Salud Publica. 2007;81:637-645.

15. García-Galicia A, Lara-Muñoz MC, Arechiga-Santamaría A, Montiel-Jarquín AJ, López-Colombo A. Validez y consistencia de una nueva escala (facial del dolor) y de la versión en español de la escala de CHEOPS para evaluar el dolor posoperatorio en niños. Cir Cir. 2012;80:510-515.

16. García-Galicia A, García-Carrasco M, Montiel-Jarquín AJ, García-Cuautitla MA, Barragán-Hervella RG, Romero-Figueroa MS. Validez y consistencia de las escalas ECAVIPEP y CAVE para evaluar la calidad de vida en pacientes pediátricos con epilepsia. Rev Neurol. 2014;59:301-306.

17. Gerónimo-Carrillo R, Guzmán-Cejas L, Magaña-Olán L, Ramos-Rendón $\mathrm{KC}$, Calidad del servicio en la consulta externa de un centro de salud urbano de Tabasco. Rev Salud Quintana Roo. 2016;9:11-15.

18. Feldman L, Vivas E, Lugli Z, Pérez MG, Bustamante S. La satisfacción del paciente hospitalario: una propuesta de evaluación. Rev Calidad Asistencial. 2007;22:133-140

19. López-Toledano MC, Luque-Cantarero I, Gómez-López VE, Casas-Cuesta R, Crespo-Montero R. La satisfacción del paciente como componente de la calidad en las unidades de diálisis. Enfer Nefrol. 2016;19:125-133.

20. Simancas-Pallares M, Arévalo-Tovar L. Desempeño de cuatro métodos estadísticos para evaluación de la concordancia prueba-reprueba de variables continuas en una muestra. Rev Biosalud. 2017;16:19-29. 